\title{
Image design of bicycle parking frame of city public facilities
}

\author{
Chao ZHAO ${ }^{1, a^{*}}$, Rui-Yu BAI ${ }^{1, b}$ and Dong ZENG ${ }^{2, c}$ \\ ${ }^{1}$ College of Art and Design, Yanshan University, Qinhuangdao, Hebei, China \\ ${ }^{2}$ Hebei Normal University of Science and Technology, Qinhuangdao, Hebei, China

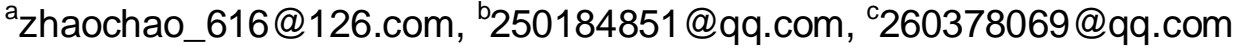 \\ ${ }^{*}$ Corresponding author
}

Keywords: Public facilities, parking frame,humanized design, structure.

\begin{abstract}
Public facilities in the bicycle parking the bicycle parking rack on the availability of re innovation of some concepts, so as to improve the bicycle frame specification Parkfunction. frame specification Parkfunction. Project team through the bicycle parking framedesign, construction of public facilities, launches the research from the bicycle parking support value, design the very pertinence and timeliness, the public facilities to better serve the people, the renovation of public facilitieshas important significance.
\end{abstract}

\section{Current situation}

With the development of the times, bicycle parking rack emerge as the times require, has become an indispensable part of life, generally divided into spiral, high-low and clamping type three (excluding type power support doesn't talk), but the bicycle parking rack on the market too old and dull, the urgent need for reform.

Bicycle parking frame every detail should carefully study design, should consider to park vehicles, indicating that the treatment, rust, tire size, economic and beautiful, the car height and other factors, many people now think that this product is simple to imitate, the results make many inappropriate parking frame.

\section{The domestic situation}

As the response to the national energy-saving emission reduction, green travel appeal, to increase people's health awareness, to bicycle travel as people will be more and more, bicycle parking frame problem is more prominent, bicycle parking rack is under this kind of background emerge as the times require. But with the development of the parking industry, the ensuing problems, generally have the following two:

The lack of innovation, product on the market is too uniform, but is spiral, slot type and level of three, and another kind of double parking frame is mainly used for display and storage, not suitable for the use of public space, so here not to mention.

Failed to fundamentally solve the basic norms of functional Park, which is mainly reflected in the bicycle parking frame for front wheel, even if the front wheel can be a very good card into the parking frame, but can not stop after the body put crooked situation, in this case, the parking frame on the body or the rear wheel can be solved very good, but also has its limitations, this type of parking frame spacing between wide Park, not suitable for intensive, but mainly in foreign countries, domestic less.

\section{The status quo abroad}

Bicycle parking frame abroad pay more attention to design and personalized appearance, this type of parking frame is more beautiful and durable, but large, which in the domestic is difficult to implement, is the first foreign population cardinal number is small, the design is more suitable for this kind of individuality, however, should fully consider the dense set of Park problem. 


\section{Trend}

The bicycle parking rack trend towards two aspects, one is the electronic development, namely, self-service bicycle park frame, the cost is high, can not be extended, but the role of cost recovery. The two is to change the appearance in the original basis, but most of the original function has not changed much, just a slightly different shape and colour.

\section{The research significance}

As everyone knows, the basic function of bicycle parking rack is mainly two kinds, one is the standard Park, the two is security, for safety, as long as the bicycle parking frame quality can be solved (here say is their lock, and bicycle parking frame with lock, the promotion is too complex, temporary not within the scope of this project), to standardize the park, as already mentioned, which is mainly reflected in the bicycle parking frame for front wheel, even if the front wheel can be a very good card into the parking frame, but not a car body put stop after crooked situation, as long as there is a bicycle crooked, then the bike is very difficult to put it, it will also have not fully regulate the park. This project is the basis for standard Park attributes of deep exploration, so as to study and design a bicycle parking frame model, try to solve this problem, and to expand the appearance and function.

\section{Research contents, project}

In this study, the main problem is the improvement of bicycle frame specification park function, then we worked out the following may occur and the key problem needed to be solved:

Bicycle frame shape and design, how to be more beautiful and practical.

On the market basically is all linear array of bicycle frame, we can design a bold vision, can be arc even bicycle parking frame around the arrangement.

If a single bicycle parking rack for individual design, how to carry on the stitching and fixing.

Taking into account the installation, disassembly when how to more convenient.

How to ensure functionality and appearance are not under the influence of the intensive, two adjacent spaces for the number of distance is the most appropriate.

To design the color, how can more easily accepted by the public.

In the original material, can re innovation, to try new material. (For example, to solve the bicycle frame longer will paint the situation)

\section{Innovations and features}

\section{Characteristics}

The project has the good practical, is aimed at the design of public facilities, and because the currently available on the bicycle frame is very simple and easy popularization, so for bicycle frame design is not much, but we want to make bold attempt on it.

\section{Innovation}

The bicycle parking rack on the market seems to have a lot, but it is not what too big difference, the innovation is that we improve from the most fundamental property, so that the bicycle parking support more perfect, and have certain extension of innovation in appearance and function, make it adapt to the development of the times. In addition, the bicycle parking support basic function and design is one of the more popular project, the project itself is a bold attempt. 


\section{The conclusion}

\section{Results (Fig. 1)}

The project team from numerous options a bicycle parking rack of a vertical, as shown below:

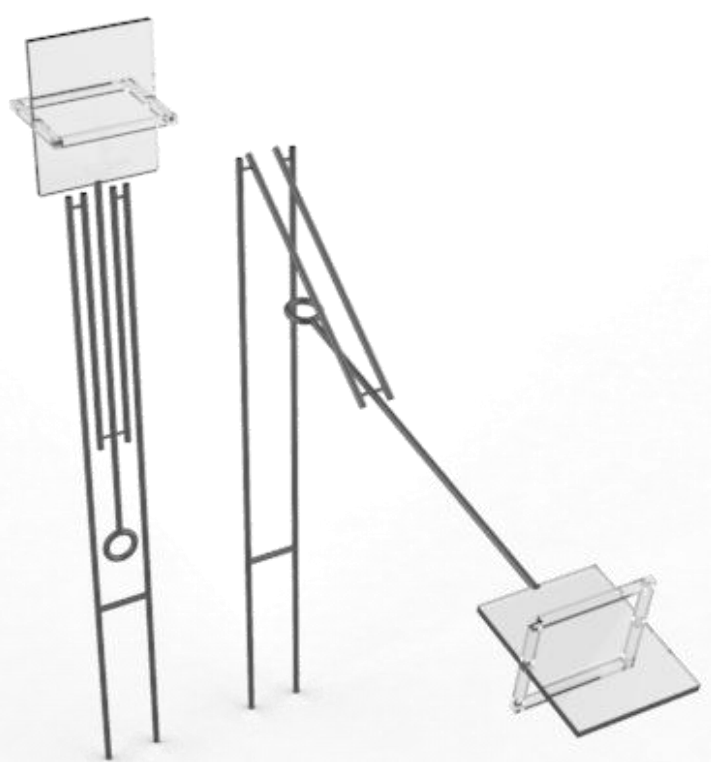

Fig. 1. Campus bicycle parking support elevation effect

\section{The working principle}

This is a semi automatic parking rack, weight below the suspension can make the upper arm back.

\section{Advantage}

To save space, the change from the traditional horizontal to vertical.

Park, not easy to appear randomly placed phenomenon,

Relatively high cost, inconvenient installation.

\section{Data generalization (Fig. 2)}

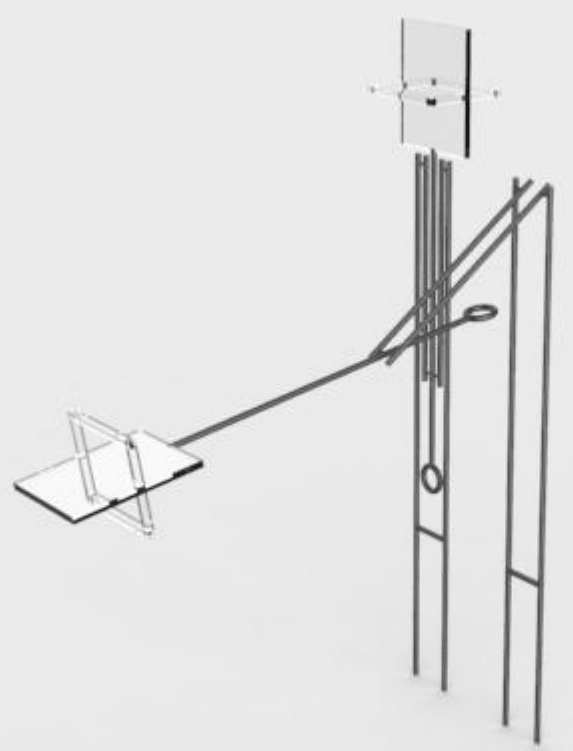

Fig. 2. Campus bicycle parking support elevation effect 
The distance between the adjacent two frame 40CM.

Wheel spacing of 10CM.

From the ground 55CM blocking wheel.

\section{Expected}

If the final product can be strong, as far as possible through commercial or other various methods of mass production, Promotion in the community, to a certain extent, change the current status of bicycle parking racks industry.

Finally, because we intend to design is to solve the problem of product specification bicycle park, if conducted a large-scale promotion of society, can solve these problems from a certain extent, to a region or a city's mental outlook has a certain degree of improvement.

\section{References}

[1]Fan Yu, Chen Bionic design [M]. Huazhong University of Science and Technology press, 2005 (11).

[2]Yuanyuan Xu bike in the overseas life [J]. tourism overview, 2012 (9).

[3]Wei Huang. The classification of electric bike [J].CHINA BICYCLE, 2012 (8).

[4]Lian Han. The energy-saving emission reduction, to explore the bike forward [J]. physics teaching high school student edition, 2009 (4).

[5]Chao Zhao. Visual guide sign of [J]. design reflects the image of the campus, 2012 (2) 